\title{
How Environmental Attitudes Interact with Cognitive Learning in a Science Lesson Module
}

\author{
Maximiliane F. Schumm and Franz X. Bogner \\ Centre of Math \& Science Education (Z-MNU), University of Bayreuth, University Campus, NW-1, 95447 Bayreuth, Germany \\ Correspondence should be addressed to Maximiliane F. Schumm; maximiliane.schumm@uni-bayreuth.de
}

Received 7 February 2016; Accepted 19 May 2016

Academic Editor: Liberato Cardellini

Copyright (C) 2016 M. F. Schumm and F. X. Bogner. This is an open access article distributed under the Creative Commons Attribution License, which permits unrestricted use, distribution, and reproduction in any medium, provided the original work is properly cited.

\begin{abstract}
As cognitive knowledge plays a major role in supporting proenvironmental behavior, identification of individual aspects related to knowledge acquisition is essential. Our study monitored knowledge levels before and after a science-based lesson set in relation to self-reported behavior and attitudinal preferences (attitudes towards environmental Preservation and Utilization) of 190 students $\left(\mathrm{M}_{\text {age }} \pm \mathrm{SD}: 15.96 \pm 0.55 ; 51.1 \%\right.$ female $)$. A knowledge questionnaire was completed once before and twice after participation. Additionally, (i) the 2-MEV (two Major Environmental Values) and (ii) the GEB (General Ecological Behavior) were applied. Girls showed higher Preservation but lower Utilization attitudes than boys did. Learning success was positively related to Preservation preferences (for girls) as well as to behavior-based scores (for girls and boys). For boys, high preferences in Utilization were negatively correlated with learning achievement.
\end{abstract}

\section{Introduction}

The energy issue is an important one in our century, although general knowledge about the meaning of energy in our lives is still quite sparse [1]. Beyond technological and scientific concerns, such as the consequences of fossil fuel exploitation or the development of energy-saving strategies, social questions need consideration (e.g., disparities between western countries and developing countries, energy supply of the future, and our impact on the earth by continuing overconsumption of energy). Science education needs to address those questions and to take into account the interaction between science, technology, and society (STS, e.g., [2]). Hodson [3] proposed enriching STS approaches with aspects of environmental education (STSE) by showing relationships to technical devices, how they are produced, and scientific principles that support understanding of our interactions with the earth's biosphere: ignoring the environmental aspect may lead to incorrect conclusions about science and technology's potential to solve (environmental) problems, for example, producing better air filters rather than diminishing air pollution. In this way, solutions to environmental problems would be ceded exclusively to experts and officials, rather than to individuals.

A concept that combines concerns of energy as crucial topic and the STSE approach is energy literacy. DeWaters and Powers [4] defined the measurable benchmarks as cognitive affective and behavioral: for example, an energy literate person is aware of energy consumption and basic energy concepts, takes responsibility for his/her energy consumption, understands the need of restriction of energy use and the development of renewable energy sources, and makes coherent personal decisions. By taking into account the abovementioned characteristics, a science-based lesson set for adolescents about renewable energies was implemented.

Cognitive Achievement. The potential of educational modules has repeatedly been demonstrated: usually, students learn cognitively during an intervention and, despite a decrease some weeks after, achievement gains persist (see, e.g., [5]). However, not all participants in those programs perform equally well in knowledge tests, since many variables may interact with achievement successes $([6,7])$. For instance, intrinsic aspects like interest $([8,9])$ or perceived self-efficacy 
[10] are supposed to relate positively to cognitive achievement. Similarly, connections between environmental attitudes and knowledge have been reported: Bogner [11] found positive relationships between knowledge and environmental perception. Dieser [12] also reported positive correlations between proenvironmental preferences and scores on a knowledge test and negative correlations with egocentric environmental attitudes and knowledge scores. In Fremerey [13], learning success was related to proenvironmental attitudes, while in Boeve-de Pauw and van Petegem [14] environmental knowledge correlated negatively with egocentric environmental preferences.

Environmental Attitudes and Behavior. A relationship of environmental attitudes with effectiveness of education modules seems to exist, although studies differ in the importance assigned to aspects of environmental attitudes related to cognitive achievement [12-14]. Measurements of attitudes and values (on the basis of 2-MEV, two Major Environmental Values) and of the reported ecological behavior (on the basis of GEB, General Ecological Behavior) have been examined $[15,16]$. Repeated independent confirmation of these models provides additional support for validity and reliability; for instance, Milfont and Duckitt [17] confirmed the existence of two attitude-set domains: the egoistic and the altruistic one (see also below). Thus, the 2-MEV with its architecture of two dimensions not only has been repeatedly confirmed by cross validation studies, for example, [18], but also has been shown to be very stable over eight subsequent cohorts (e.g., [19]). The two dimensions are an anthropocentric perspective reflecting the Utilization of natural resources and a more biocentric perspective with a focus on protection and conservation of natural resources. Preservation reveals rather selfless protection and conservation preferences towards the natural environment. Utilization in contrast addresses the use of natural resources and environment with the respondent as the main beneficiary [19]. This model thus allows the assessment of two practically independent dimensions. That means that assessment of the estimated importance of making use of natural resources does not conflict with assessment of high environmental protection values. The $2-\mathrm{MEV}$ is especially designed for adolescents. Its performance has been repeatedly independently confirmed (e.g., [14, 20, 21]).

Proenvironmental behavior focuses on the reduction of negative effects of actions on the natural world, by reducing, for example, waste production or consumption of resources [22]. Despite the fact that a person with a proenvironmental attitude may show proenvironmental behavior, the direct relation between attitude and behavior is controversial: a person, for example, holding environmental attitudes should not eat meat, in order to minimize her/his ecological footprint; another person may not eat meat for other reasons. We cannot say that this person also has a preserving environmental attitude because other motives may lie behind the non-meateating behavior, for example, special diet for health reasons. Nevertheless, Kaiser et al. [16] argue that when monitoring $a$ set of behaviors, as the GEB does, the underlying attitude can be extracted quite well.
Sex-Specific Differences in Environmental Attitudes. Differences in environmental attitudes have frequently been described in the literature (e.g., [23]). Females are often reported to have higher proenvironmental values and demonstrate more proenvironmental behavior than males do. Those differences may originate in socialization processes and gender roles (e.g., [24]), as in many cultures women are expected to be cooperative, helpful, and attentive whereas men are thought to be aligned with autonomy and competitive orientation [25]. Stern et al. [26] reported women as being more sensitive to the consequences of interference with the ecosystem affecting other species and the biosphere and holding stronger biospheric-altruistic values. Fremerey [13], for instance, reported for girls higher Preservation but lower Utilization attitude sets. In contrast, Boeve-de Pauw and van Petegem [14] reported higher utilitarian preferences in males but no higher proenvironmental attitudes in females. Also, Dieser [12] found no gender differences in the environmental attitudes of children. Similar to the relation between cognitive achievement and environmental attitudes (see above), the pattern structure of gender differences in environmental attitudes seems complex. Therefore, these two issues may need further disentangling efforts. Consequently, our research questions are as follows:

(i) Can we find sex-specific differences in Preservation and Utilization as well as in the reported behavior scores?

(ii) Do correlations of environmental attitudes and reported behavior with cognitive achievement differ for boys and girls?

\section{Material and Methods}

2.1. Participants. Our sample consisted of 232 students $\left(\mathrm{M}_{\text {age }} \pm \mathrm{SD}: 16.02 \pm 0.56 ; 50.41 \%\right.$ female $)$ of 10 th-grade college preparatory school ("Gymnasium"). Gymnasium is a school type of advanced secondary education. It focuses on academic learning to prepare adolescents for studying at a university. It leads to higher education entrance qualification. Pupils who perform well in primary school or lower secondary school are permitted to pass over to a "Gymnasium," mostly in the age between 10 and 11. Depending on the performance, students continue "Gymnasium" until grade 12 or 13, aged between 17 and 19. Teachers enrolled their classes for a learning module with associated data collection and parents approved the participation of their children by signing a declaration of consent. In total, eleven classes (average number of students per class: 21) of five schools in northern Bavaria participated in our study.

2.2. Science-Based Environmental Education Module. A three-lesson module (135 minutes) about the use of fossil fuels and alternative energy sources was applied [5]. The intention of our didactic materials was to support interdisciplinary learning on different topics (e.g., global warming: chemistry of burning fossil fuels, physics of the greenhouse effect, and ecological effects of the latter). In this way, we combined science with environmental education [3] to 
present a holistic picture of the issue. The topics conformed to the 10th-grade curriculum.

To support cooperative learning, participants worked in pairs. Guidance was given by a workbook, and the order of learning stations was chosen autonomously. Two teachers were available as guides. Sample solutions for each work station were available on demand at the teachers' desk.

2.2.1. Test Design. An ad hoc knowledge questionnaire was applied accompanied by (i) the 2-MEV to measure "two Major Environmental Values" [15] and (ii) the GEB to measure self-reported "General Ecological Behavior" [16]. The knowledge questionnaire was administered three times to track individual knowledge development. The 2-MEV and the GEB were administered once.

Knowledge about specific environmental and scientific aspects around renewable energies was assessed by a multiple-choice test (e.g., "What would be the consequence if there would be no natural greenhouse effect?"; "How can we avoid dinner plate or fuel tank conflict?"; "What kind of power plant generates the highest electricity yields per year?"; "Which domains have to be taken into account for the change in energy supply to be sustainable?" [5]). Each of the 21 items was provided with four possible answers with one correct answer. The knowledge questionnaire was designed according to the learning module and was pilottested with students as well as experts before application. The questionnaire was administered one week before $(T 0)$, directly after (T1), and six weeks after (T2) participation in the learning module. To avoid test effects, the order of items and distractors were changed for each test schedule. Students were not informed about any testing schedule, to prevent undesired preparation.

The 2-MEV questionnaire [15] was applied in its modified version of Kibbe et al. [27]. Due to time restrictions in the application of the questionnaire, only 10 items of the 20 were selected (on the basis of the factor loadings plotted in Kibbe et al. [27] and of the content of the items). As the 2-MEV measures two domains labeled Preservation (PRES) and Utilization (UTIL), 5 items of each subscale were chosen (see Table 1). Responses were elicited via a five-point Likert scale from strongly disagree (1) to strongly agree (5). Behaviorbased preferences were collected with the GEB, specially designed for adolescents [16]. The 40 original items of the GEB are grouped into the six domains recycling, waste avoidance, consumerism, mobility and transport, energy conservation, and vicarious conservation behaviors. As according to Kaiser et al. [16] one subdomain can already predict any other one, we selected 14 items that best matched the content of the learning module (domain energy conservation, 6 items, e.g." "After one day of use, my sweaters and trousers go into the laundry"; domain mobility and transport, 3 items, e.g., "I ride a bicycle, take public transportation or walk to school"; 5 selected items of the domain vicarious behaviors towards conservation, e.g., "I insist on holidays close to home"). The response format was a five-point Likert scale from totally incorrect (1) to totally correct (5).
TABLE 1: Exploratory factor analysis for the 2-MEV for 10 items, $N=$ 190.

\begin{tabular}{lcc}
\hline Items & PRES & UTIL \\
\hline $\begin{array}{l}\text { 1PP: It upsets me to see the countryside taken over } \\
\text { by building sites. }\end{array}$ & .782 & \\
7PP: Dirty industrial smokes from chimneys & .700 & \\
make me angry. & & \\
15UN: Humans do not have the right to change & .592 & \\
nature as they see fit. & & \\
9PP: It is interesting to know what kind of & .566 & \\
creatures live in ponds or rivers. & & \\
18UN: Human beings are not more important & .434 & \\
than other creatures. & & .736 \\
17UP: We must build more roads so people can & & .689 \\
travel to the countryside. & & .568 \\
19UP: People worry too much about pollution. & & \\
16UP: We need to clear forests in order to grow & & .556 \\
crops. & & .384 \\
3PN: We don't need to set aside areas to protect \\
endangered species.
\end{tabular}

2.2.2. Statistical Analysis. For statistical analysis, SPSS (Version 22.0) was used. Complete datasets of 190 students $\left(\mathrm{M}_{\text {age }} \pm \mathrm{SD}: 15.96 \pm 0.55 ; 51.1 \%\right.$ female $)$ were considered for our analysis. The following scores have been calculated for each student: mean scores for Preservation and Utilization preferences and the reported behavior and sum score means for the knowledge questionnaire. Following the central limit theorem, normal distribution was assumed, and therefore parametric testing was used.

For the 2-MEV, factor structure of the 10 items was extracted by a principal component factor analysis with oblique rotation (direct oblimin) with the number of factors fixed to two. In the next step, using the independent $t$-test, gender differences were examined in the two 2-MEV domains (UTIL and PRES) and in the GEB.

In preparation for the next step, answers of the knowledge questionnaire were recoded to 1 for correct and 0 for incorrect. Individual test scores were calculated by summing the single answer scores of the 21 questions. To examine the relations between 2-MEV and GEB with knowledge in T0, T1, and T2, a two-tailed Pearson correlation separately for boys and girls was performed and Bonferroni correction employed.

\section{Results}

A principal component factor analysis confirmed the twofactor structure of the 2-MEV, although we had applied a shortened version. Table 1 shows the factors after oblique rotation (factor 1 reflecting Preservation and factor 2 reflecting Utilization of nature). This confirms the structure of the instrument to measure both attitude sets. The KMO value of 


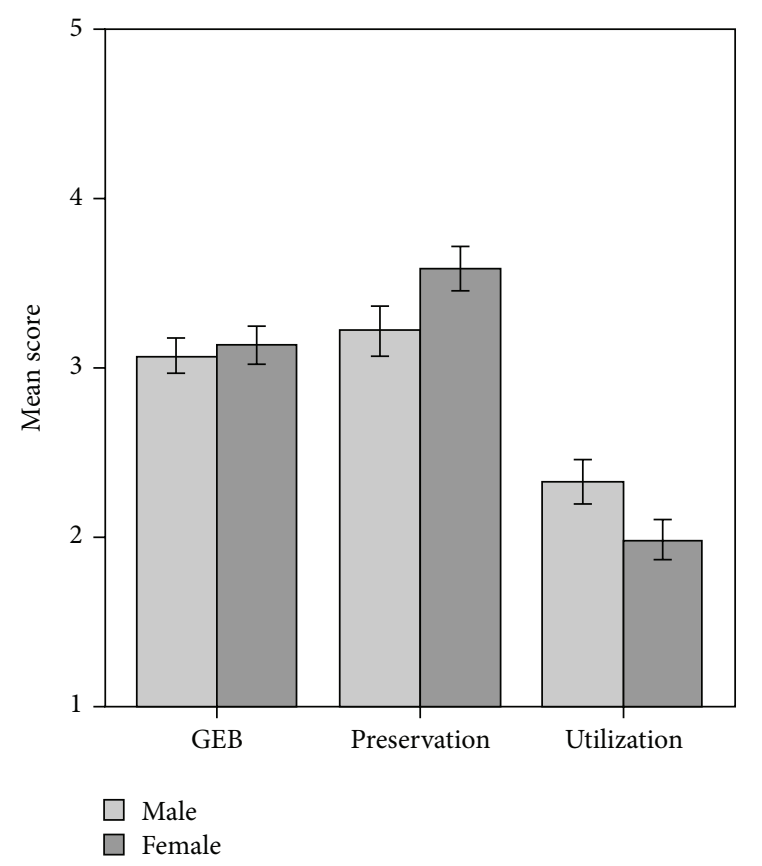

Figure 1: Gender differences are only observable for the 2-MEV domains; $N=190$; error bars show 95\% CI.

0.65 was sufficient to verify the sampling adequacy. Also all KMO scores of the individual items were above the critical value of 0.5 [28]. No cross-loading exceeded 0.3.

An independent $t$-test showed males $(\mathrm{M} \pm \mathrm{SD}=2.32 \pm$ .61) as scoring significantly higher on Exploitative Utilization than females $(\mathrm{M} \pm \mathrm{SD}=1.99 \pm .57)$. The difference $(0.33$, $95 \%$ CI $[0.17,0.51])$ is significant $(t(188)=3.95, p<.001)$ with a nearly medium effect size $(r=0.28)$. The reverse was observed for Preservation: females $(\mathrm{M} \pm \mathrm{SD}=3.58 \pm .67)$ yield higher scores than males $(\mathrm{M} \pm \mathrm{SD}=3.22 \pm .72)$. This difference $(-0.37,95 \%$ CI $[-0.57,-0.17])$ is also significant $(t(188)=-3.67, p<.001, r=0.26)$. In contrast, in the GEB, no gender differences occurred (Figure 1).

With Bonferroni correction applied, neither environmental attitudes nor behavior correlated with knowledge in the pretest, but they did in both retests (Table 2). PRES and GEB showed positive relations with retest knowledge, whereas UTIL is negatively related to knowledge scores $T 1$ and $T 2$. For females, correlations between knowledge and PRES and GEB are evident for $T 1$ and $T 2$, whereas knowledge of male participants correlates with UTIL and GEB only at T2. All significant correlations showed medium effect size.

\section{Discussion}

First of all, the solid structure of the $2-\mathrm{MEV}$, even with a reduced number and reverse phrased items, again appeared in its clear two-dimensionality. This is not surprising because its consistency has been confirmed in several independent studies (e.g., [17, 20, 21]). Recently, Borchers et al. [20] showed for the 2-MEV scale the stability of a 16-item version, instead of the full 20-item set, monitored over a period of eight years. Also cross-sectional studies with different populations
TABLE 2: Correlations between knowledge scores with 2-MEV and GEB, Bonferroni correction ${ }^{*} p_{\text {sig }} \leq .003$. PRES: Preservation; UTIL: Exploitative Utilization; m: male $(n=93)$; f: female $(n=97)$.

\begin{tabular}{cccccccc}
\hline \multirow{2}{*}{ Knowledge } & \multicolumn{2}{c}{ T0 } & \multicolumn{2}{c}{$T 1$} & \multicolumn{2}{c}{$T 2$} \\
& $\mathrm{~m}$ & $\mathrm{f}$ & $\mathrm{m}$ & $\mathrm{f}$ & $\mathrm{m}$ & $\mathrm{f}$ \\
\hline PRES & & & & & & \\
$r$ & .068 & .050 & .111 &. $\mathbf{3 6 5}$ & .222 &. $\mathbf{3 0 4}$ \\
$p$ & .519 & .625 & .288 & $<.001$ & .033 & .002 \\
UTIL & & & & & & \\
$r$ & -.219 & -.035 & -.281 & -.259 & $-.444^{*}$ & -.170 \\
$p$ & .035 & .730 & .006 & .010 & $<.001$ & .095 \\
GEB & & & & & & \\
$r$ &. $\mathbf{1 6 2}$ & .288 & .253 & $.357^{*}$ &. $\mathbf{3 0 9}$ & $.358^{*}$ \\
$p$ & .121 & .004 & .014 & $<.001$ & .003 & $<.001$ \\
\hline & & & & & &
\end{tabular}

confirmed the 2-MEV model of two underlying variables even with less than the full 20 items (e.g., [12]). One advantage of a reduced item set is its better applicability in limited time frames of intervention studies.

Like in Bogner and Wiseman ([15], subjects' age about 14) or Fremerey ([13], subjects' age about 12), girls showed higher preserving but lower Utilization attitudes than boys. This dichotomous pattern is not consistent, with other studies finding a different one: Boeve-de Pauw and van Petegem ([14], subjects about 11 years old) reported higher utilitarian preferences for males but found no higher proenvironmental attitudes for females. Dieser ([12], subjects about 10 years old) found no sex-specific differences of the two MEV domains at all. Nevertheless, if differences do appear, females tend to score higher on proenvironmental attitudes. A commonly used explanation for the rather proenvironmental attitudes of women [24] is that they may react more sensitively to the consequences for species or the biosphere, thus obtaining stronger biospheric-altruistic values [26].

Another explanation for sex-specific effects of the 2-MEV is reported by Boeve-de Pauw et al. [29] who discussed decoding differences of item wordings. Our study, however, used an optimized version of the $2-\mathrm{MEV}$ with modified wording of some items [27], which may have overcome such objections. Also, proenvironmental results of women caused by social desirability issues could be attenuated for the same reason: the negative wording of some items of the applied 2-MEV scale should prevent the effects of social desirability from interfering [27]. Nevertheless, repeating the study of Oerke and Bogner [30] for the adjusted version of the 2MEV would be advisable to see if the negatively coded items counteract the influence of social desirability as predicted.

No sex-specific differences are evident for the selfreported ecological behavior. Accordingly, both 2-MEV and GEB showed different gender sensitivity. Proenvironmental attitudes may not result in proenvironmental behavior, as difficulties or social hurdle may hinder a person from acting in a proenvironmental way despite attitudinal prerequisites (e.g., $[16,21])$. One consequence of this argumentation is that the behavior-based approach of the GEB may prevent the detection of sex-specific differences. That may imply that 
the GEB and the MEV are measuring different aspects of environmental preferences. Nevertheless, the meta-analysis of Zelezny et al. [24] pointed out for most of the included studies sex-specific preferences with respect to proenvironmental behavior $\left(r_{\text {gender }}=0.1\right)$, even more than for attitude $\left(r_{\text {gender }}=0.07\right)$.

Knowledge levels in the pretest (T0) did not correlate with environmental attitudes for boys and for girls, though this is no longer the case for the retest scores after module participation: with girls, a positive Preservation score implied positive knowledge scores in both retests; for boys, a utilitarian attitude was detrimental to their long-term learning success. The fact that correlations were observed only for the retests suggests that attitudes are related to learning but not to previous knowledge. Girls' learning seems to be positively influenced by high Preservation scores but not by low Utilization scores. In contrast, for boys, low Utilization scores seem to be essential for effective learning.

With a similar test design, Dieser [12] reported correlations of knowledge scores with Utilization already in the pretest; in retest 1 and retest 2, the correlation was observed for both Preservation and Utilization scores; however, the reported effects are smaller than in our study (highest correlation knowledge with UTIL $=-.298$, with PRES = .181). Consequently, low Utilization scorers already started with less previous knowledge. Dieser [12] saw previous knowledge as connected to environmental attitudes, whereas in our study clearly learning was positively linked to high Preservation preferences (for girls) and even more to low Exploitative Utilization preferences (for boys). We presume that among others age made the difference: subjects in Dieser's [12] study were fourth and fifth graders (aged about 10 years). An additional explanation might originate in the knowledge item design: while Dieser [12] focused on environmental and biological knowledge ("Why were animal bridges built?"; "What is a young lynx doing after leaving his mother"), we asked more for science-based environmental issues ("What would be the consequence if there would be no natural greenhouse effect?"; "How can we avoid dinner plate or fuel tank conflict?" [5]). Children with a positive attitude towards nature might also have more environmental knowledge independently of an educational intervention. In contrast, students positively disposed to environmental issues who participated in our study might be more motivated to learn even if they had no knowledge advantages in the pretest. Boeve-de Pauw and van Petegem [14] reported for subjects about 11 years old environmental knowledge as negatively correlated with Utilization but not with Preservation attitudes. In contrast, Fremerey [13] found significant correlations only with knowledge in Preservation scores (subjects about 12 years old). Reasons for the varying results on this issue may lie in the different educational backgrounds the studies are based on. Furthermore, our study dealt with adolescents, whereas the studies cited above monitored children aged between 10 and 12 years.

In conclusion, we showed learning success and Preservation attitudes (for girls) to be positively related. As in the literature, knowledge is consistently described as one influential factor of environmental attitudes (e.g., [21]); we can presume for students with higher knowledge levels a more ecocentric worldview and, in turn, higher proenvironmental attitudes to interact with their learning. In this way, a cycle of interacting factors (knowledge-proenvironmental attitudes) may develop. Moreover, especially for boys, high preferences in Utilization showed a negative impact on learning. That negative environmental values could prevent learning is also described by Kollmuss and Agyeman [22]. Our findings suggest that environmental education programs should respond to this gender specific issue as different aspects for boys and girls seem decisive for learning.

The correlations between GEB scores and knowledge are similar for boys and girls. It should be noticed that for boys the correlation first appeared in the second retest while for girls the effects were already evident directly after the intervention. One reason for this phenomenon may be that boys with low and high Utilization and GEB scores at first learned equally effectively but boys with higher Utilization scores and lower GEB scores showed worse recall abilities. Before we continue to discuss the relations of the GEB with knowledge scores, we need to emphasize that this discussion is only reasonable if we see the GEB as described by Kaiser et al. [16]: as an instrument to quantify self-reported behavior with a strong connection to attitudes behind the reported behavior. There is scant literature comparing the relation of reported behavior measured with the GEB with achievement like our study. In the competence model for environmental education $[31,32]$, too, the GEB does not influence knowledge dimensions. Nevertheless, the connection between GEB and learning could be explained by the following. (i) Subjects with environmentally friendly behavior preferences may show higher individual interest in the topic energy consumption today and in the future [33]. (ii) Options for individual actions were especially integrated into the learning module and were part of the knowledge questionnaire (see [5]). In this way, students with self-reported ecofriendly behavior may also have been more open to new behavior patterns addressed during the intervention. (iii) Moreover, as mentioned above, Kaiser et al. [16] postulated for the GEB a substantial overlap with the preserving and Utilization attitudes.

\section{Conclusion}

As many people even in the knowledge-based societies cannot answer questions about, for example, generation of usable energy, personal consumption, or saving of energy [1], educational efforts in energy issues in schools and universities should be prioritized. Aguirre-Bielschowsky et al. [34] highlighted a need for stimulation of conversation about energy at school and at home to achieve responsible citizenship. To improve knowledge situations, appropriate learning modules combining all aspects of energy literacy (cognitive, affective, and behavioral) are needed [4]. In our study, environmental attitudes are differently related to learning achievement of boys and girls. Instructors should take into account individual differences by applying and offering diverse learning environments. As for boys low Utilization and for girls high Preservation preferences frequently seem to exist (e.g., [15]) learning environments addressing requirements of both 
sexes need specific consideration. Nevertheless, as knowledge acquisition and individual changes towards more ecocentric preferences always portray the germ of any outreach educational initiatives, educators may even better complete their intent when variables intervening with these frames are better known. The issue of energy education surely provides a suitable vehicle to reach these goals.

\section{Disclosure}

Any opinions, findings, conclusions, or recommendations expressed in this material are those of the authors and do not necessarily reflect the position of the University of Bayreuth or the European Commission.

\section{Competing Interests}

The authors declare that there are no competing interests regarding the publication of this article. Also, the mentioned received grants, scholarships, and funding do not lead to any competing interests regarding the publication of this manuscript.

\section{Acknowledgments}

This study was supported by the University of Bayreuth and the Inspiring Science Education project funded by the European Union's ICT Policy Support Program as part of the Competitiveness and Innovation Framework Program (no. 32512). The authors would like to thank Michael Wiseman for his feedback on an early version of this manuscript.

\section{References}

[1] N. Armaroli and V. Balzani, "The future of energy supply: challenges and opportunities," Angewandte Chemie-International Edition, vol. 46, no. 1-2, pp. 52-66, 2007.

[2] G. S. Aikenhead, "STS education: a rose by any other name," in A Vision for Science Education: Responding to the Work of Peter Fensham, pp. 59-75, RoutledgeFalmer, New York, NY, USA, 2003.

[3] D. Hodson, "Time for action: science education for an alternative future," International Journal of Science Education, vol. 25, no. 6, pp. 645-670, 2003.

[4] J. DeWaters and S. Powers, "Establishing measurement criteria for an energy literacy questionnaire," Journal of Environmental Education, vol. 44, no. 1, pp. 38-55, 2013.

[5] M. F. Schumm and F. X. Bogner, "The impact of science motivation on cognitive achievement within a 3-lesson unit about renewable energies," Studies in Educational Evaluation, vol. 50, pp. 14-21, 2016.

[6] J. Hattie, Visible Learning. A Synthesis of Over 800 Meta-Analyses Relating to Achievement, Routledge, London, UK, 2009.

[7] C. Randler, E. Hummel, M. Gläser-Zikuda, C. Vollmer, F. X. Bogner, and P. Mayring, "Reliability and validation of a short scale to measure situational emotions in science education," International Journal of Environmental and Science Education, vol. 6, no. 4, pp. 359-370, 2011.
[8] M. F. Schumm and F. X. Bogner, "Measuring adolescent science motivation," International Journal of Science Education, vol. 38, no. 3, pp. 434-449, 2016.

[9] D. H. Schunk, P. R. Pintrich, and J. L. Meece, Motivation in Education. Theory, Research, and Applications, Pearson/Merrill Prentice Hall, Upper Saddle River, NJ, USA, 3rd edition, 2008.

[10] F. Pajares, "Gender and perceived self-efficacy in self-regulated learning," Theory into Practice, vol. 41, no. 2, pp. 116-125, 2002.

[11] F. X. Bogner, "The influence of short-term outdoor ecology education on long-term variables of environmental perspective," The Journal of Environmental Education, vol. 29, no. 4, pp. 1729, 1998.

[12] O. Dieser, Kognitive leistungen, einstellungen und assoziationen: eine erlebnisorientierte interventionsstudie am außerschulischen Lernort Nationalpark [Ph.D. dissertation], University of Bayreuth, Bayreuth, Germany, 2015, https://epub.uni-bayreuth .de/2072/.

[13] C. Fremerey, Forschend-entdeckendes Lernen zum Thema Trinkwasser am außerschulischen Lernort [Ph.D. dissertation], University of Bayreuth, Bayreuth, Germany, 2015, https://epub .uni-bayreuth.de/1812/.

[14] J. Boeve-de Pauw and P. van Petegem, "The effect of Flemish eco-schools on student environmental knowledge, attitudes, and affect," International Journal of Science Education, vol. 33, no. 11, pp. 1513-1538, 2011.

[15] F. X. Bogner and M. Wiseman, "Adolescents' attitudes towards nature and environment: quantifying the 2-MEV model," Environmentalist, vol. 26, no. 4, pp. 247-254, 2006.

[16] F. G. Kaiser, B. Oerke, and F. X. Bogner, "Behavior-based environmental attitude: development of an instrument for adolescents," Journal of Environmental Psychology, vol. 27, no. 3, pp. 242-251, 2007.

[17] T. L. Milfont and J. Duckitt, "The structure of environmental attitudes: a first- and second-order confirmatory factor analysis," Journal of Environmental Psychology, vol. 24, no. 3, pp. 289303, 2004.

[18] F. X. Bogner and M. Wiseman, "Environmental perception of rural and urban pupils," Journal of Environmental Psychology, vol. 17, no. 2, pp. 111-122, 1997.

[19] F. X. Bogner, B. Johnson, S. Buxner, and L. Felix, "The 2-MEV model: constancy of adolescent environmental values within an 8-year time frame," International Journal of Science Education, vol. 37, no. 12, pp. 1938-1952, 2015.

[20] C. Borchers, C. Boesch, J. Riedel, H. Guilahoux, D. Ouattara, and C. Randler, "Environmental education in côte d'ivoire/West Africa: extra-curricular primary school teaching shows positive impact on environmental knowledge and attitudes," International Journal of Science Education, vol. 4, no. 3, pp. 240-259, 2013.

[21] B. Johnson and C. C. Manoli, "Using bogner and Wiseman's model of ecological values to measure the impact of an earth education programme on children's environmental perceptions," Environmental Education Research, vol. 14, no. 2, pp. 115$127,2008$.

[22] A. Kollmuss and J. Agyeman, "Mind the gap: why do people act environmentally and what are the barriers to proenvironmental behavior?" Environmental Education Research, vol. 8, no. 3, pp. 239-260, 2002.

[23] J. Boeve-de Pauw, V. Donche, and P. van Petegem, "Adolescents' environmental worldview and personality: an explorative study," Journal of Environmental Psychology, vol. 31, no. 2, pp. 109-117, 2011. 
[24] L. C. Zelezny, P.-P. Chua, and C. Aldrich, "Elaborating on gender differences in environmentalism," Journal of Social Issues, vol. 56, no. 3, pp. 443-457, 2000.

[25] A. H. Eagly and M. E. Kite, "Are stereotypes of nationalities applied to both women and men?" Journal of Personality and Social Psychology, vol. 53, no. 3, pp. 451-462, 1987.

[26] P. C. Stern, L. Kalof, T. Dietz, and G. A. Guagnano, "Values, beliefs, and proenvironmental action: attitude formation toward emergent attitude objects," Journal of Applied Social Psychology, vol. 25, no. 18, pp. 1611-1636, 1995.

[27] A. Kibbe, F. X. Bogner, and F. G. Kaiser, "Exploitative vs. appreciative use of nature-two interpretations of utilization and their relevance for environmental education," Studies in Educational Evaluation, vol. 41, pp. 106-112, 2014.

[28] A. Field, Discovering Statistics Using IBM SPSS Statistics: And Sex and Drugs and Rock ' $n$ ' Roll, 4th edition, 2013.

[29] J. Boeve-de Pauw, K. Jacobs, and P. van Petegem, "Gender differences in environmental values: an issue of measurement?" Environment and Behavior, vol. 46, no. 3, pp. 373-397, 2014.

[30] B. Oerke and F. X. Bogner, "Social desirability, environmental attitudes, and general ecological behaviour in children," International Journal of Science Education, vol. 35, no. 5, pp. 713-730, 2013.

[31] F. G. Kaiser, N. Roczen, and F. X. Bogner, "Competence formation in environmental education: advancing ecology-specific rather than general abilities," Umweltpsychologie, vol. 12, no. 2, pp. 56-70, 2008.

[32] N. Roczen, F. G. Kaiser, F. X. Bogner, and M. Wilson, "A competence model for environmental education," Environment and Behavior, vol. 46, no. 8, pp. 972-992, 2013.

[33] U. Schiefele, "Interest, learning, and motivation," Educational Psychologist, vol. 26, no. 3-4, pp. 299-323, 1991.

[34] I. Aguirre-Bielschowsky, R. Lawson, J. Stephenson, and S. Todd, "Energy literacy and agency of New Zealand children," Environmental Education Research, 2015. 


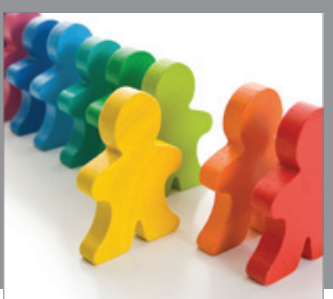

Autism

Research and Treatment
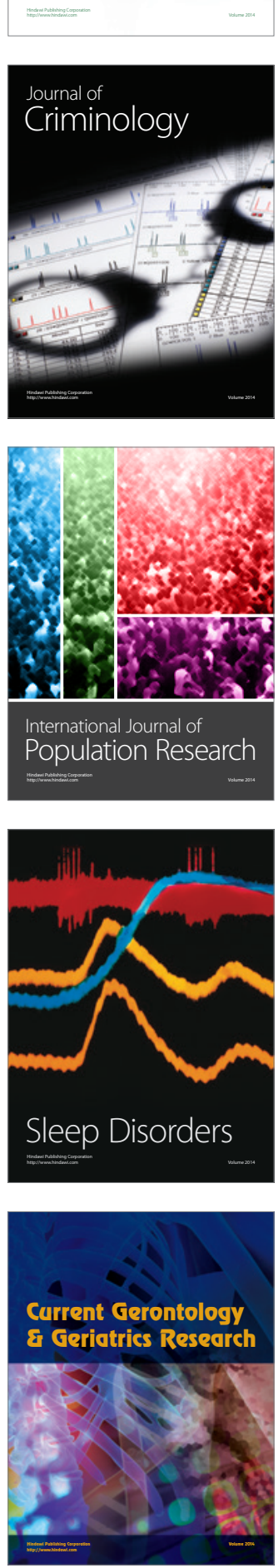

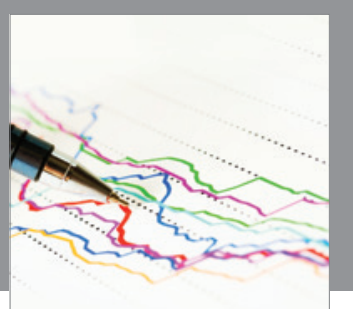

Economics

Research International
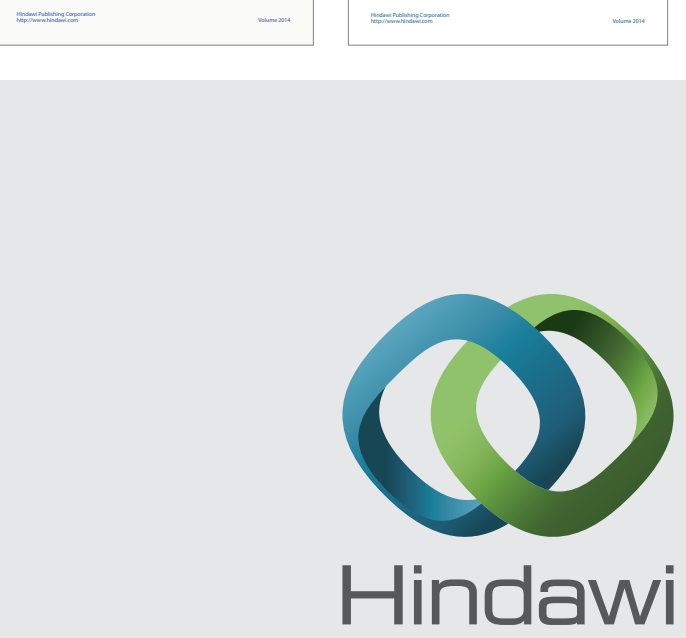

Submit your manuscripts at

http://www.hindawi.com
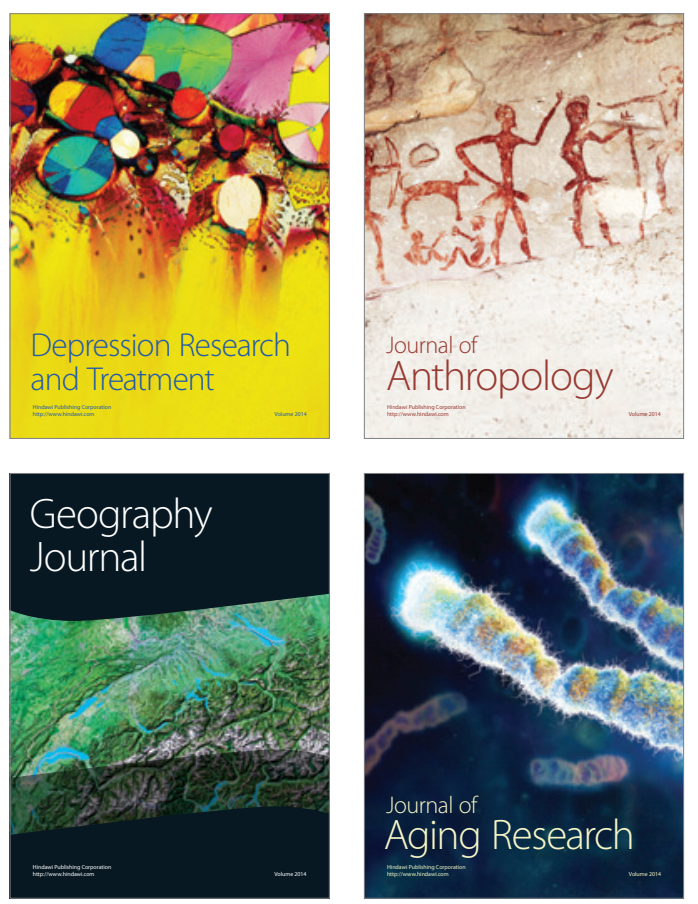
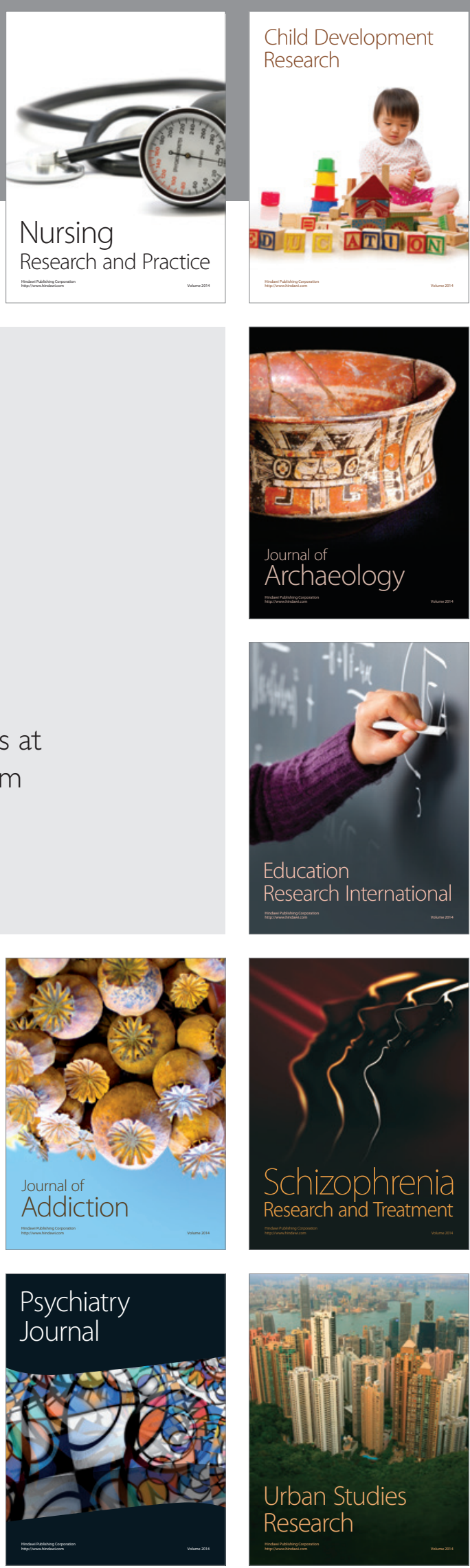